Linguistique, littérature, didactique

151-152 | 2011

Anthropologies de la littérature

\title{
« Il n'y a jamais que des contextes »
}

Les communautés interprétatives de Stanley Fish

\section{Raymond Michel}

\section{OpenEdition}

Journals

Édition électronique

URL : http://journals.openedition.org/pratiques/1777

DOI : 10.4000/pratiques. 1777

ISSN : 2425-2042

Éditeur

Centre de recherche sur les médiations (CREM)

Édition imprimée

Date de publication : 15 décembre 2011

Pagination : 49-72

\section{Référence électronique}

Raymond Michel, « « II n'y a jamais que des contextes » », Pratiques [En ligne], 151-152 | 2011, mis en ligne le 16 juin 2014, consulté le 14 novembre 2019. URL : http://journals.openedition.org/pratiques/ 1777 ; DOI : 10.4000/pratiques. 1777 


\title{
"II n'y a jamais que des contextes ${ }^{(1)}$ » Les communautés interprétatives de Stanley Fish
}

\author{
Raymond Michel \\ CELTED, Université Paul Verlaine - Metz
}

\begin{abstract}
« Découvrir le sens de l'œuvre, sens plus ou moins caché et qui est assigné à des niveaux divers ${ }^{(2)}{ }$ tel est le but, ordinaire et communément admis, que se donne la critique littéraire. En conformité avec ce présupposé, les pratiques critiques, dans la tradition universitaire, se déclinent - cum grano salis et très schématiquement, car les enchevêtrements ne sont pas rares - en trois grandes familles : la critique historique, la critique herméneutique, la critique formelle. Elles sont toutes fondées sur les mêmes postulats épistémologiques : le texte possède des propriétés (structurelles, linguistiques, stylistiques, énonciatives, thématiques, etc.) essentielles, objectives, pérennes et autonomes par rapport au regard critique. Ainsi, la critique historique tente de remonter vers un hors texte qui serait doté d'un pouvoir explicatif et causal; par exemple, le contexte - social, politique, culturel entre autres - de production et de réception de l'œuvre pour l'histoire littéraire ; le secret d'une vie pour l'approche biographique. Les critiques herméneutiques (psychanalytique, thématique...) sont, quant à elles, à la recherche de la « clé » de l'œuvre, qui déterminerait sa signification, clé qui se dissimulerait dans les propriétés linguistiques et sémantiques du texte. Enfin, la critique formelle vise à dégager, à un niveau micro et/ou macro-textuel, le système du texte, en postulant, généralement, comme le faisait le structuralisme, l'immanence du sens et la clôture du texte. Ces approches sont redevables d'une conception essentialiste de la connaissance et d'une modélisation verticale et « correspondantiste » de la "vérité », définie comme l'adéquation de la description aux propriétés, objectives et autonomes, de l'objet étudié. Et, donc, dans ce cas comme le fait très bien remarquer Roland Barthes :
\end{abstract}

L'œuvre se ferme sur un signifié. On peut attribuer à ce signifié deux modes de signification : ou bien on le prétend apparent, et l'œuvre est alors l'objet d'une science de la lettre qui est la philologie ; ou bien ce signifié est réputé secret, dernier, il faut le chercher, et l'œuvre relève alors d'une herméneutique, d'une interprétation (marxiste, psychanalytique, thématique, etc.) $;[\ldots]^{(3)}$.

(1) Richard Rorty, Objectivisme, relativisme et vérité, 1991, trad. de l'anglais (américain) par J.-P. Cometti, Paris, PUF, coll. «L'Interrogation philosophique »1994, p. 115.

(2) Roland Barthes, «Texte (théorie du) », Encyclopcedia Universalis, 1975.

(3) Roland Barthes, Le Bruissement de la langue, Paris, Éd. du Seuil, 1993, p. 74. 
C'est avec une telle conception, dominante dans les études littéraires, que Stanley Fish veut rompre, et ce avec éclat.

\section{«Une bombe à retardement »}

Toutefois, la doxa ayant force de loi, il apparaît que la traduction du «petit » livre de Stanley Fish, Quand lire c'est faire. L'autorité des communautés interprétatives ${ }^{(4)}$, n'a pas eu le retentissement qu'il méritait dans la réflexion qui se mène dans le champ de la théorie littéraire française ${ }^{(5)}$. Je rappellerai que ce volume contient les textes suivants : «Y a-t-il un texte dans ce cours? », "Comment reconnaître un poème quand on en voit un », « Démonstration vs. Persuasion : deux modèles d'activité critique », et « Folger Papers ». L'éditeur précise, fort à propos, le contexte des interventions de Stanley Fish :

Les trois premiers essais qui composent ce livre trouvent leur double origine dans l'incident qui a donné lieu au premier d'entre eux et dans l'article de Meyer Abrams, "How To Do Things With Texts", une attaque frontale du travail de Jacques Derrida, Harold Blomm, et Stanley Fish. Ils consistaient initialement en une série de conférences prononcées au Kenyion College du 8 au 13 avril 1979 dans le cadre du John Crowe Ransom Memorial Lecture. Ils ont ensuite pris place dans le livre Is There a Text in this Class? The Authority of Interpretive Communities (Harvard University Press). Quant au quatrième essai, il est tiré de Professional Correctness : Literary Studies and Political Change (Oxford University Press, 1995) ${ }^{(6)}$.

On peut se demander pourquoi il a fallu près de vingt sept ans pour traduire en français ces textes, et ce dans une « petite» maison d'édition militante, «Les Prairies ordinaires ». Il est vrai que Stanley Fish, perçu comme un affabulateur scandaleux, n'est pas très connu en France, du moins il n'aurait pas très bonne presse. Tout d'abord, on a eu tendance à l'assimiler à son avatar romanesque avoué. En effet, David Lodge, dans Changement de décor et dans Un tout petit monde ${ }^{(7)}$, se serait servi de lui pour créer son personnage, Moris Zapp, un universitaire et un théoricien de la littérature renommé. Ce spécialiste de Jane Austin, qui enseigne dans une université américaine prestigieuse et qui vit dans une maison de rêve, est bien peu sympathique tant il est excentrique, ambitieux, imbu de sa personne, arrogant et sûr de lui-même à l'excès, et souvent condescendant avec ses collègues et ses potentiels adversaires. De plus, si l'on peut dire, il est obsédé par l'argent et le sexe. Aussi Marc Escola a-t-il raison de rappeler que Stanley Fish a pu apparaître « dans la réalité comme dans la fiction, [comme] "le professeur de littérature le mieux payé de la planète" (le premier sinon le seul "littéraire" à bénéficier d'un salaire annuel à six chiffres en dollars...) et l'homme de toutes les polémiques sur les

(4) Stanley Fish, Quand lire c'est faire. L'autorité des communautés interprétatives, trad. de l'anglais (américain) par Étienne Dobenesque, préface d'Yves Citton, postface inédite de Stanley Fish, Paris, Les Prairies ordinaires, coll. «Penser/Croiser», 2007, 144 p. ; dorénavant abrégé en $Q L F$.

(5) Il faut signaler, toutefois, deux articles très éclairants mis en ligne, qui ont nourri cette réflexion : Marc Escola, «L'autorité de l'interprète. Les fables théoriques de Stanley Fish », publié surFabula, «Acta », 2008, URL : http ://www.fabula.org/revue/document3780.php ; Frank Wagner, « Actualité(s) de Stanley Fish », publié sur Vox-poetica "Articles », 2009, URL : http ://www.vox-poetica.org/t/articles/wagner2009.html. Consultés le 20 juin 2011.

(6) QLF., p. 28

(7) David Lodge a, en fait,, écrit une trilogie satirique sur le monde universitaire et l'homo academicus : Changing Places, 1975, Small World, An Academic Romance, 1984, et Nice Work, 1988, parus chez Martin Secker \& Warburg Ltd ; ces livres ont été traduits de l'anglais en français par Maurice et Yvonne Couturier sous les titres respectifs Changement de décor, 1991, Un tout petit monde, 1992, et Jeu de société, 1991, tous trois publiés par Payot \& Rivages, coll. « Rivages poche/Bibliothèque étrangère ». 
questions de politique universitaire comme sur les sujets les plus brûlants du débat public nord-américain ${ }^{(8)} \gg-$ la discrimination positive, la liberté d'expression et ses limites, la rhétorique conservatrice, les politiques universitaires, etc. Ensuite, d'un point de vue plus scientifique, il faut remarquer qu'il n'existait qu'un seul ouvrage de Stanley Fish traduit en français en 1998, Respecter le sens commun. Rhétorique, interprétation et critique en littérature et en droit ${ }^{(9)}$. La présentation, sur la quatrième de couverture, de l'auteur par son éditeur français avait essentiellement pour cible des lecteurs spécialisés en droit :

Stanley Fish est un des acteurs les plus importants du mouvement de « Droit et Littérature », qui est un des courants majeurs dans la théorie américaine du droit. Professeur de Littérature anglaise, Professeur de Droit, Vice-Recteur associé, et Directeur exécutif des Presses universitaires à la Duke University (États-Unis), il a publié largement sur la prose et la poésie de la période de la Renaissance en Angleterre, sur la théorie de la littérature et du droit, sur la liberté d'expression, et sur la structure et le fonctionnement disciplinaires.

On peut comprendre que ce livre, publié dans une collection intitulée « Pensée juridique moderne », ait eu si peu d'écho en France auprès des « littéraires », même s'il a été minutieusement commenté par Jacques Derrida dans un essai percutant, « Du droit à la justice $^{(10)} »$. En fait, comme le remarque Marc Escola, les thèses de Stanley Fish sur l'interprétation des textes littéraires n'étaient accessibles que par l'entremise des « exposés assassins de ses plus ardents détracteurs ${ }^{(11)}$ ", à savoir en particulier Umberto Eco dans Les Limites de l'interprétation et Antoine Compagnon dans Le Démon de la théo$r i e^{(12)}$, ce dernier n'hésitant pas de parler de «gnose » à propos des positions théoriques de Stanley Fish qu'il range sous l'étiquette de « relativiste dogmatique ». Et pourtant, à la suite de Marc Escola, on peut considérer Stanley Fish comme «l'un des plus grands théoriciens littéraires du second $\mathrm{XX}^{\mathrm{e}}$ siècle, à l'égal de Barthes et de Derrida ${ }^{(13)}$ ", même s'il n'a cessé de susciter par ses écrits la polémique. Il faut donc lire Stanley Fish, cette « bombe à retardement ${ }^{(14)}$ », comme le préconise Yves Citton, dans sa préface.

Le titre français du recueil fait évidemment référence à un des ouvrages les plus célèbres de J.-L. Austin, Quand dire, c'est faire ${ }^{(15)}$ et inscrit donc la réflexion de Stanley Fish dans la tradition du pragmatisme nord-américain représentée par John Dewey, William James ou Richard Rorty, pour nommer les plus connus en France. Il n'en reste pas moins, comme le fait remarquer Marc Escola, que :

Plus fidèle à l'original anglais, le sous-titre «L'autorité des communautés interprétatives » (The Autority of Interpretive Communities) nomme sans doute mieux le propos

(8) Marc Escola, «L'autorité de l'interprète. Les fables théoriques de Stanley Fish », art. cit.

(9) Stanley Fish, Doing What Comes Naturally: Change, Rhetoric, and The Practice of Theory in Literary and Legal Studies, Durham, NC: Duke UP, 1989, trad. de 1'anglais (américain) par Odile de Nerhot, Penser le sens commun : Rhétorique, interprétation et critique en littérature et en droit, $\mathrm{Pa}-$ ris, Story Scientia-Droit, L.G.D.J, coll. «Pensée juridique moderne », 1998.

(10) Jacques Derrida, « Du droit à la justice», in Force de loi. Le Fondement mystique de l'autorité, Paris, Galilée, coll. « La Philosophie en effet», 1994.

(11) Marc Escola, «L'autorité de l'interprète. Les fables théoriques de Stanley Fish », art. cit.

(12) Umberto Eco, Les Limites de l'interprétation, 1990, trad. de l'italien par Myriem Bouzaher, Paris, Grasset, 1992 ; Antoine Compagnon, Le Démon de la théorie. Littérature et sens commun, Paris, Éd. du Seuil, coll. « La couleur des idées », 1998.

(13) Marc Escola, art. cit.

(14) $Q L F$., Yves Citton, "Puissance des communautés interprétatives », p. 16.

(15) John Langshaw Austin, How to do things with Words: The William James Lectures delivered at Harvard University in 1955, Éd. Urmson, Oxford, 1962, trad. de 1'anglais par Gilles Lane, Quand dire, c'est faire, Paris, Éd. du Seuil, 1970, rééd. Coll. « Points essais », 1992. 
de l'ensemble et la principale originalité de l'ouvrage pour la théorie littéraire ; la question qu'affronte S. Fish, à la fin des années soixante-dix donc, est bien celle du statut de l'interprétation, de l'autorité du texte en regard de celle de l'interprète et de ce qu' on pourrait nommer le «partage des significations». On peut énoncer cette question dans les termes simples, ou « continentaux », d'une alternative brutale : doit-on penser que le sens d'un texte se confond avec la signification qu'un auteur a en lui déposé et qu'il s'agit donc pour le suffisant interprète de mettre au jour ou de « retrouver » (mais alors comment expliquer la diversité des interprétations dont ce même texte peut faire historiquement l'objet ?) ; ou bien : un texte peut-il recevoir tous les sens qu'il nous plaît de lui donner (mais peut-on alors accepter qu'aucune interprétation puisse être dite plus juste qu'une autre ?). Pour l'énoncer autrement : quelles sont donc les autorités respectives de l'auteur et de l'interprète ${ }^{(16)}$ ?

Pour répondre à ces questions Stanley Fish invente, comme l'indique Pascal NicolasLe Strat, un personnage conceptuel ${ }^{(17)}$, le lecteur-faiseur de textes, c'est-à-dire un lecteur qui n'interprète pas un texte, mais le fabrique. C'est ce personnage qu'il met en scène dans une fable théorique, "Comment reconnaître un poème quand on en voit un $^{(18)} »$. Pour éviter d'assimiler cette thèse à un slogan provocateur et sophistique, il est nécessaire de suivre de près la déconstruction que mène Stanley Fish.

\section{Une fable expérimentale}

Dans son essai «Comment reconnaître un poème quand on en voit un » Stanley Fish raconte une anecdote qui illustre sa thèse essentielle : «les significations ne sont la propriété ni de textes stables et fixes ni de lecteurs libres et indépendants, mais de communautés interprétatives qui sont responsables à la fois de la forme des activités d'un lecteur et des textes que cette activité produit ${ }^{(19)}$. "

Pendant l'été 1971, Stanley Fish donne, deux cours dans la même salle. À 9h30, il rencontre des étudiants avec qui il examine, dans une perspective théorique, les présupposés et les préconceptions qui sous-tendent à la fois la linguistique et la critique littéraire. À 11 h00, ces étudiants sont remplacés par de «purs » littéraires, avec qui il étudie la poésie religieuse anglaise du $17^{\mathrm{e}}$; ces derniers ont déjà appris avec lui « comment identifier les symboles chrétiens et reconnaître des schèmes typologiques, et comment passer de l'observation de ces symboles et modèles à la spécification d'une intention poétique qui était généralement d'ordre didactique ou sermonnaire ${ }^{(20)}$. » Stanley Fish avait donné comme devoir à ses premiers étudiants la lecture de quelques linguistes, tous spécialistes de la Grammaire Générative et Transformationnelle, qu'il jugeait essentiels et dont il avait écrit la liste des noms au tableau ; liste qu'il n'avait pas effacée quand le deuxième groupe d'étudiants l'avait rejoint pour suivre son cours de littérature.

La liste était disposée verticalement, et les noms étaient plus ou moins centrés, d'une façon tout à fait fortuite, par rapport à la paire Roderick Jacobs et Peter Rosenbaum auteurs de manuels et d'anthologie bien connus. Le nom d'Ohman était suivi d'un point d'interrogation car Stanley Fish ne se souvenait plus s'il prenait un ou deux «n». Entre

(16) Marc Escola, art. cit.

(17) Pascal Nicolas-Le-Strat, «Un usager faiseur de textes », in Moments de l'expérimentation, Montpellier, Fulenn Éditions, 2009.

(18) QLF., pp. 55-77.

(19) Ibid., p. 55 ; je souligne.

(20) Ibid., p. 56. 
les deux cours, le professeur n'avait procédé qu'à deux changements : il avait tracé un cadre autour de la liste et écrit au-dessus du cadre «p. $43 »$. Soit l'inscription suivante :

p. 43

\begin{tabular}{|c|}
\hline Jacobs-Rosenbaum \\
Levin \\
Thorne \\
Hayes \\
Ohman (?) \\
\hline
\end{tabular}

Stanley Fish indique aux étudiants de son second cours que ce qu'ils voient au tableau est un poème religieux du type de ceux qu'ils ont déjà étudiés. Il leur demande de l'interpréter; et, note Stanley Fish, « immédiatement, ils s'exécutèrent (perform) d'une façon qui [...] était plus ou moins prévisible ${ }^{(21)} »$. Les étudiants rivalisent d'imagination et d'ingéniosité ; Stanley Fish énumère les différentes interprétations de ses étudiants qui « dévoilent» le sens religieux de chaque mot et trouvent une cohérence textuelle et théologique à la liste des noms inscrits au tableau. Sans entrer dans les détails, mais pour en donner une idée, j'en énumère quelques éléments : la forme du "poème " est interprétée comme un hiéroglyphe qui représenterait une croix ou un autel ; le premier « vers » est vu comme faisant référence à l'échelle de Jacob, représentation allégorique de l'ascension chrétienne vers le Ciel, grâce à l'intercession de la Vierge Marie, présente métonymiquement par le symbole du rosier-Rosenbaum -, et elle-même considérée comme une " rose sans épines », emblème de l'Immaculée conception; « Levin » ferait référence à la fois à la tribu de Levi dont la fonction sacerdotale a été accomplie par le Christ et au pain sans levain (leaven) emporté par les enfants d'Israël lors de l'Exode hors de l'Égypte, lieu du péché, suite à l'appel de Moïse, modèle du Christ dans 1'Ancien Testament; « thorne» ferait allusion à la couronne d'épines dont on avait affublé le Christ lors de la Passion, prix à payer pour le salut des hommes; le dernier mot du poème est l'objet de trois lectures complémentaires : comme « omen » (présage), puisque une grande partie du poème se présenterait comme une prophétie, comme «Oh Man », puisque le sujet du poème serait l'histoire de l'homme en ses lieux de croisement avec le plan du divin, et comme «Amen », «juste conclusion du poème célébrant l'amour et la miséricorde de Dieu, qui a donné son Fils unique pour que nous vivions ${ }^{(22)} »$.

Mais les étudiants de Stanley Fish n’en restent pas là, car « après avoir spécifié et mis en rapport les sens à donner aux mots du poème, [ils] commencèrent à discerner des schèmes structuraux plus vastes ${ }^{(23)} »$. Ils font valoir que parmi les six noms présents trois sont hébreux (Jacobs, Rosenbaum, Levin), deux chrétiens (Thorn, Hayes), le dernier demeurant ambigu, ambiguïté signalée par le point d'interrogation mis entre parenthèses. Cette opposition vétéro/néotestamentaire reflète la distinction fondamentale entre l'ancien et le nouveau testament. Enfin, les plus astucieux, font remarquer que les lettres les plus fréquentes réitérées dans le poème sont les lettres « $S$ », « $O$ » et « $\mathrm{N} »$, dont la coalescence renvoie au mot «Son»-le «Fils»-, c'est-à-dire au Christ, le Fils de l'Homme qui a réalisé la prophétie de la venue du Messie proclamée par l'Ancien Testament.

En fait, pour Stanley Fish il s'agit moins d'exposer l'ingéniosité de ses étudiants que de se saisir de ce récit pour s'interroger sur leurcapacité à se prêter à un tel exercice :

(21) Ibid., p. 57.

(22) Ibid., p. 59.

(23) Ibid. 
Quelle est la source de cette capacité ? Comment se fait-il qu'ils aient pu faire ce qu'ils ont fait? Ces questions sont importantes puisqu'elles se rapportent directement à une question récurrente de la théorie littéraire : quelles sont les marques distinctives du langage littéraire ? Ou, pour parler plus familièrement : comment reconnaissez-vous un poème quand vous en voyez un ${ }^{(24)}$ ?

\section{Un double déplacement}

L'exploitation théorique qu'il fait de cette fable, comme le rappelle Yves Citton dans sa préface, permet à Stanley Fish d'effectuer un double déplacement : dans un premier temps, il établit le pouvoir créatif du lecteur ; puis, dans un deuxième temps, il rapporte ce pouvoir au contexte, dans lequel exerce le lecteur-faiseur de texte.

\section{Le pouvoir créatif du lecteur}

Tout d'abord, Stanley Fish rompt avec le sens commun et la doxa dominante dans les études des textes (des linguistes aux stylisticiens en passant par les «purs » littéraires) pour qui il va de soi que la signification est déposée dans un texte et qu'elle peu être trouvée et reconstituée par la discrimination et l'analyse de ses propriétés, jugées pertinentes, repérables sur les plans de la forme du contenu et de l'expression. Mais un tel modèle ne convient pas à ce qui s'est passé dans la classe de Stanley Fish, car ses étudiants

[...] ne sont pas passés de l'observation de marques distinctives à la reconnaissance qu'ils faisaient face à un poème; au contraire, c'est l'acte de reconnaissance qui fut premier - ils savaient à l'avance qu'ils avaient affaire à un poème - et les marques distinctives ont suivi. [...] En d'autres termes, loin d'être provoqués par des caractéristiques formelles, les actes de reconnaissance sont leur source. Ce n'est pas la présence de qualités poétiques qui impose un certain type d'attention mais c'est le fait de prêter un certain type d'attention qui conduit à l'émergence de qualités poétiques ${ }^{(25)}$.

Dit autrement, c'est parce que les étudiants étaient persuadés que ce qu'ils voyaient au tableau était un poème, qu'ils « commencèrent à regarder avec des yeux "qui voient de la poésie", c'est-à-dire avec des yeux qui voient tout en relation avec les propriétés qu'ils savent que les poèmes possèdent ${ }^{(26)}$. » En effet, on peut présumer que Stanley Fish leur a appris qu'un poème possède « une organisation plus dense et plus complexe que les communications ordinaires ${ }^{(27)} »$, et donc, forts de cette compétence, ils ont été tout à fait disposés « à voir des connexions entre un mot et un autre et entre tous les mots et la perspective d'ensemble du poème ${ }^{(28)}$. » Ils ont cherché à fonder - et ont réussi, audelà de toute attente - une isotopie totalisante du " poème », qui guidait la résolution du sens de chaque mot, lequel confirmait l'hypothèse globale, selon la logique interprétative spécifique et inhérente au « cercle herméneutique ». Et Stanley Fish de conclure, non sans malice :

C'était presque comme s'ils suivaient une recette de cuisine - s'il s'agit d'un poème, faites ceci, s'il s'agit d'un poème, voyez-le de cette manière - et de fait, les définitions de la poésie sont des recettes, puisqu'en imposant aux lecteurs ce qu'ils doivent cher-

(24) Ibid., p. 60.

(25) Ibid., je souligne.

(26) Ibid.

(27) Ibid., p. 61.

(28) Ibid. 
cher dans un poème, elles leur enseignent des manières de regarder qui produisent ce qu'ils s'attendent à voir. Si votre définition de la poésie vous dit que le langage de la poésie est complexe, vous allez sonder le langage de cette chose identifiée à un poème de manière à faire ressortir la complexité que vous savez être « là » ${ }^{(29)}$.

Ainsi un lecteur de poésie sera attentif à la présence d'ambiguïtés latentes, de séries allitératives et assonancées, de significations divergentes par rapport à l'opinion commune, etc. Et en faisant cela, il n'aura «pas l'impression d'agir (performing) en forçant le texte, puisqu' [il] ne fera que ce qu'[il] aura appris en devenant un lecteur de poésie compétent ${ }^{(30)}$. » Et donc constate Stanley Fish :

La compétence de lecture est généralement conçue comme une capacité à discerner ce qui est là, mais si l'exemple de mes étudiants peut être généralisé, c'est une capacité à savoir comment produire ce dont on peut dire, après coup, qu'il est là. L'interprétation n'est pas l'art d'analyser (construing) mais l'art de construire (constructing). Les interprètes ne décodent pas les poèmes : ils les font (they make them $)^{(31)}$.

Lire, pour reprendre une expression de Michel de Certeau ${ }^{(32)}$, est donc un art de faire, qui se caractérise par « la capacité à fabriquer le texte le plus approprié au contexte institutionnel dans lequel le lecteur évolue et le plus en phase avec la situation dans laquelle il agit ${ }^{(33)} »$. Il faudra y revenir.

Bien évidemment, une telle conclusion - «pénible », pour reprendre la qualificatif de Stanley Fish lui-même, tant elle heurte le sens commun pour ne pas dire le bon sens -, n'est pas sans soulever de nombreuses objections auxquelles le théoricien ne manque pas de répondre avec beaucoup d'aplomb. Tout d'abord, on peut faire remarquer que ses étudiants étaient conditionnés par le contexte de leur cours qui portait précisément sur la poésie religieuse et donc vulnérables à la tromperie organisée par leur professeur et enclins, par confiance ou soumission, à imposer des significations religieuses aux mots qui leur étaient présentés ; mais Stanley Fish affirme qu'il a répété l'expérience des dizaines de fois, dans des universités différentes, dans des pays différente, et que les résultats ont toujours été les mêmes. On peut, aussi, objecter que la liste des noms soumise à la sagacité des interprètes se prête à une telle interprétation par un heureux hasard et qu'il n'en serait pas de même avec des noms moins connotés bibliquement ; Stanley Fish refuse une telle hypothèse, car il est convaincu que ses étudiants auraient été capables de transformer n'importe quelle liste de noms en poème religieux, dans la mesure où ils auraient lu ces noms à l'intérieur du présupposé qu'ils étaient informés par un sens chrétien; aussi prend-il soin de préciser qu'il a répété l'expérience avec une liste prise au hasard - celle des noms de ses collègues - et a pu constater que les lecteurs sollicités ont «fait le poème » sans difficulté particulière. D'ailleurs, précise-t-il, il ne faudrait pas restreindre cette créativité à la lecture "poétique », car assimiler l'énumération des noms à un « devoir », ou même à une simple « liste », nécessite un travail d'interprétation aussi élaboré que pour produire un poème. En effet, les étudiants de Stanley Fish ne voient pas d'abord un objet primaire, neutre et autonome - une liste - qu'ils interprètent ensuite comme un « devoir à faire » ou un " poème ». Le fait de voir une liste, même si l'évidence de l'habitude et la pression du contexte universitaire tend à nous le faire oublier, mobilise aussi des compétences acquises, des connaissances tacites et un

(29) Ibid., pp. 61-62; souligné par l'auteur.

(30) Ibid., p. 62.

(31) Ibid. ; souligné par 1'auteur.

(32) Michel de Certeau, L'Invention du quotidien, 1. Arts de faire et 2. Habiter, cuisiner, éd. établie et présentée par Luce Giard, Paris, Gallimard, 1990.

(33) Pascal Nicolas-Le Strat, «Un usager faiseur de textes », op. cit., p. 130. 
système d'intelligibilité complexe ${ }^{(34)}$. Cela revient à dire, selon Stanley Fish, ce que chacun sait en fait, même s'il a tendance à l'oublier :

[...] les poèmes et les sujets de devoir sont différents, mais j'insiste sur le fait que ces différences sont le résultat des opérations interprétatives différentes que nous réalisons et non de quelque chose qui serait inhérent à un poème ou à sujet de devoir. Un sujet de devoir n'impose pas davantage sa propre reconnaissance qu'un poème ; comme dans le cas d'un poème, la forme d'un sujet de devoir émerge lorsque quelqu'un regarde cette chose identifiée à un sujet de devoir avec des jeux « qui voient un sujet de devoir », c'est-à-dire avec des yeux qui sont capables de voir les mots comme déjà inclus à l'intérieur de la structure institutionnelle qui fait qu'il est possible que les sujets de devoir aient un sens. La capacité à voir, et donc à faire, un sujet de devoir, n'est pas moins acquise que la capacité à voir, et donc à faire, un poème. L'un comme l'autre sont des objets construits, produits, et non producteurs, de l'interprétation, et si les différences entre les deux sont bel et bien réelles, elles sont interprétatives et n'ont pas leur source dans quelque premier niveau objectif ${ }^{(35)}$.

Puisque le sujet et l'objet sont indissolublement liés, cognitivement parlant, il faut admettre qu'il n'existe pas d'objet indépendant d'un sujet immergé dans un contexte d'intelligibilité et que tout objet est fait par les stratégies que le sujet met en œuvre et non pas trouvé.

On peut imaginer aisément les sourires mi- moqueurs et mi-scandalisés qu'une telle position a pu et peut encore provoquer. Au mieux, on y voit une provocation insignifiante et un goût pour le sophisme et la fumisterie, non dénuée de perversité, qui font le « charme » de Stanley Fish ; au pire, on dénonce tous les maux qu'une telle attitude théorique peut entraîner : accepter tous les délires (sur)interprétatifs les plus cocasses ; donner un blanc-seing au n'importe quoi énoncé par n'importe qui ; rendre caduc tout enseignement et toute évaluation puisque toutes les lectures se valent et qu'aucune n'est vraie ou fausse ; enfermer chaque lecteur dans le solipsisme le plus complet et le plus laxiste ; miner toute rigueur méthodologique herméneutique et faire fi des sciences du texte ; ignorer les travaux menés en histoire littéraire et philologie ; sombrer dans le relativisme philosophique, puisqu'est affirmée l'impossibilité d'une connaissance objective et non-projective ; mettre en danger les études littéraires et la rigueur de pensée ; dénigrer l'esprit scientifique, etc. On peut se demander, à juste titre, si ces critiques s'adressent réellement aux travaux de Stanley Fish, tant elles paraissent ne prendre en compte qu'un aspect - le plus « scandaleux »? - de sa thèse, en " oubliant» la suite de son raisonnement.

\section{L'empire des communautés interprétatives}

En effet, après avoir dépouillé le texte de son autonomie et de tout pouvoir préalable à son interprétation, après avoir exemplifié le pouvoir créatif du lecteur-faiseur de textes, Stanley Fish opère un deuxième déplacement : il reverse ce pouvoir aux communautés interprétatives dans lesquelles est quasiment embrigadé tout lecteur. Effectivement, la mise en avant du pouvoir créatif du lecteur, contrairement à ce qu'ont proclamé, avec une belle unanimité, les détracteurs de Stanley Fish, n'aboutit pas, chez lui, à l'affirmation et à la célébration d'un subjectivisme et d'un relativisme effrénés. Dans tous ses essais, Stanley Fish insiste sur le fait que nous ne sommes pas condamnés à la subjectivité, dans la mesure où les moyens par lesquels nous interprétons/voyons les objets

(34) Telle est aussi la thèse défendue dans le premier essai, "Y a-t-il un texte dans le cours ? , $Q L F$., pp. 29-53.

(35) Ibid., p. 67 
comme ceci ou comme cela sont sociaux et conventionnels. Le sujet qui fabrique un poème ou un devoir est un sujet communautaire, et non un individu isolé, dont les opérations mentales sont "limitées par les institutions dans lesquelles [il est] déjà inclus ${ }^{(36)}$. » Stanley Fish est catégorique :

Ces institutions nous précèdent, et c'est seulement en les habitant, ou en étant habités par elles, que nous avons accès aux sens publics et conventionnels qu'elles produisent. Ainsi, s'il est vrai que nous créons la poésie (et les sujets de devoirs et les listes), nous la créons au moyen de stratégies interprétatives qui ne sont finalement pas les nôtres, mais qui ont leur source dans un système d'intelligibilité de disponibilité publique ${ }^{(37)}$.

Ainsi, pour rester dans ce domaine, le système littéraire « nous contraint, il nous façonne également, en nous munissant de catégories de compréhension avec lesquelles nous façonnons à notre tour les entités que nous pouvons alors désigner ${ }^{(38)}$. " Tout comme les poèmes, les listes ou les sujets de devoirs, nous sommes des objets faits ou construits, car nous sommes " les produits de schèmes de pensées sociaux et cultur rels ${ }^{(39)}$. » Stanley Fish est donc amené à refuser l'opposition entre objectivité et subjectivité, « puisque ni l'une ni l'autre n'existe dans la forme pure qui donnerait sa valeur à l'opposition ${ }^{(40)}$. »C C'est ce qu'exemplifie l'anecdote qu'il a racontée :

[elle] montre que nous n'avons pas affaire à des lecteurs autonomes en relation de perception adéquate ou inadéquate avec un texte tout aussi autonome. Au contraire, nous avons affaire à des lecteurs dont les consciences sont constituées par un ensemble de notions conventionnelles qui, une fois mises en marche, constituent à leur tour un objet conventionnel et vu conventionnellement. Mes étudiants ont pu faire ce qu'ils ont fait, et ils l'on fait de concert, parce qu'en tant que membres de la communauté littéraire, ils savaient ce qu'était un poème, et cette connaissance les a conduits à regarder le paysage de manière à le peupler de ce qu'ils savaient être des poèmes ${ }^{(41)}$.

Il est donc erroné d'imaginer que les étudiants de Stanley Fish auraient d'abord perçu des traces inscrites au tableau, traces purement physiques, objectives et pré-interprétatives, et qu'ils les auraient, ensuite, interprétées comme une "liste de noms », ou un " devoir à faire", ou un "poème». Ils donnent du sens «par le fait de voir, et non après avoir vu ${ }^{(42)}$. » La thèse est sans appel : nous voyons toujours d'une certaine manière, nous sommes toujours plongés dans un contexte, dont nous avons incorporé et appris les intérêts, les objectifs et les usages, et dans une situation qui nous conduit à «voir $x$ comme $y$ ». En fait, il faudrait préciser que, si le sujet construit l'objet, à son tour et dans le même mouvement, l'objet construit le sujet : je fais le poème et le poème me fait lecteur de poème. Mais, rappelons-le :

[...] la manière de voir, quelle qu'elle soit, ne serait jamais individuelle ou idiosyncrasique, puisque sa source est la structure institutionnelle dont le « voyant » est l'agent et l'extension. [...] une culture remplit les cerveaux [...] au point que nul ne peut dire que ses actes interprétatifs lui sont absolument propres mais qu'ils lui échoient en vertu de sa position dans un environnement organisé et qu'ils sont donc toujours publics et partagés ${ }^{(43)}$.

(36) Ibid. ; souligné par 1'auteur.

(37) Ibid., pp. 68-69.

(38) Ibid., p. 69.

(39) Ibid.

(40) Ibid.

(41) Ibid.; je souligne.

(42) Ibid., p. 72 ; souligné par l'auteur.

(43) Ibid., p. 73. 
La crainte des partisans de l'interprétation objective - pour qui les textes portent en eux une signification déjà là, autonome et indépendante de son lecteur chargé de la dévoiler - est donc sans objet et sans pertinence. Il n'existe pas de sujet - un ego-non contraint (the unconstrained self) et solipsiste qui projetterait ses préjugés, sinon ses fantasmes, sur un texte, «car l'ego n'existe pas en dehors des catégories de pensée conventionnelles et communautaires qui habilitent ses opérations (lire, penser, voir) ${ }^{(44)}$. » Il s'ensuit que :

[...] si l'ego est conçu, non comme une entité indépendante mais comme une construction sociale dont les opérations sont délimitées par les systèmes d'intelligibilité qui l'informent, alors les significations qu'il confère au texte ne sont pas les siennes mais trouvent leur source dans la (ou les) communauté (s) interprétative (s) sur laquelle (lesquelles) il repose ${ }^{(45)}$.

Ces significations, précise Stanley Fish, ne sont ni objectives ni subjectives; elles ne sont pas objectives « parce que elles sont toujours le produit d'un point de vue plutôt que simplement "lues" "; elles ne sont pas subjectives "parce que ce point de vue sera toujours social ou institutionnel ${ }^{(46)} »$. Elles sont donc « à la fois subjectives et objectives : elles sont subjectives parce qu'inhérentes à un point de vue particulier et donc non universelles; et elles sont objectives parce que le point de vue qui les délivre est public et conventionnel plutôt qu'individuel ou singulier ${ }^{(47)}$.» En effet, Stanley Fish, en pragmatiste conséquent, comme on l'a vu, rejette toute disjonction ontologique entre d'une part les interprètes (toujours susceptibles d'oublier le texte), et d'autre part les textes (toujours susceptibles d'imposer ses dictats). Car «si les ego sont constitués par les manières de penser et de voir inhérentes aux organisations sociales, et si, à leur tour, ces ego constitués constituent des textes selon ces mêmes manières, il ne peut donc y avoir de rapport d'antagonisme entre le texte et l'ego puisqu'ils sont les produits nécessairement liés des mêmes possibilités cognitives ${ }^{(48)}$. » Et donc il est tout aussi exact de dire que les lecteurs font les significations ou que « les significations, sous la forme de catégories interprétatives issues de la culture, font les lecteurs ${ }^{(49)}$. »

On le voit, les thèses de Stanley Fish provoquent un double effondrement : celui de l'autonomie du texte et celui de l'autonomie du lecteur. En effet, les protocoles d'interprétation ne sont ni fixés et enfouis dans le texte, ni confiés à la liberté, éventuellement sauvage, d'un lecteur souverain et solipsiste. Bien au contraire, les lecteurs sont strictement contraints dans leurs activités interprétatives par les règles - les recettes, dirait Stanley Fish - intériorisées de la communauté interprétative à laquelle ils appartiennent, communauté qui fonctionne comme un système d'intelligibilité et comme un guide d'actions partagés qui ne laissent aucune liberté au lecteur. Antoine Compagnon a très bien identifié, à cet égard, l'ultraïsme des thèses de Stanley Fish qui entérinent, d'une certaine façon, l'anéantissement de la liberté du lecteur et l'expulsion de sa subjectivité, qui sont pourtant admises, ordinairement, comme consubstantielles à l'acte même de la lecture ${ }^{(50)}$. C'est ce qui le distingue de l'École de Constance et qui explique le hourvari avec lequel ses analyses ont été souvent accueillies dans le landernau littéraire :

(44) Ibid.

(45) Ibid., p. 74 ; je souligne.

(46) Ibid.

(47) Ibid.; souligné par l'auteur.

(48) Ibid., p. 75.

(49) Ibid.

(50) On peut opposer à la vision de Stanley Fish celle de Marielle Macé, par exemple, qui défend l'idée d'une expérience idiosyncrasique et subjective de la lecture, dans Façons de lire, manières d'être, Paris, Gallimard, coll. « NRF Essais », 2011. 
Ces communautés interprétatives, à la manière du répertoire d'Iser ou de l'horizon d'attente de Jauss, sont des ensembles de normes d'interprétation, littéraires et extralittéraires, qu'un groupe partage : des conventions, un code, une idéologie, si l'on veut. Mais, à la différence du répertoire et de l'horizon d'attente, la communauté interprétative ne laisse plus la moindre autonomie au lecteur, ou plus exactement à la lecture, ni au texte qui résulte de la lecture : avec le jeu de la norme et de l'écart, toute subjectivité est désormais abolie ${ }^{(51)}$.

Il n'existe donc pas, pour Stanley Fish, de signification " hors sol », qui, toujours déjà là, survivrait à une transformation de contexte et de situation. Si un texte, déjà interprété, est reçu, synchroniquement ou diachroniquement, dans une nouvelle communauté interprétative, sa signification se recompose, se réagence et s'inscrit dans un nouvel horizon de sens et de pratiques.

\section{Des communautés interprétatives aux communautés d'usage}

Il est incontestable que la réflexion de Stanley Fish constitue une ouverture théorique tout à fait passionnante, tant elle déplace le «lieu » de la signification et tant elle prend à rebours le sens commun et les certitudes les plus établies. Toutefois, on peut regretter, avec Pascal Nicolas-Le Strat, la façon dont il s'emploie à la « refermer, patiemment et consciencieusement ${ }^{(52)}$. » En effet, selon ce dernier, la question de la portée et de la puissance instituante et constituante des communautés interprétatives est relativement délaissée « au profit d'un rappel insistant du caractère contraignant et limitatif de ces communautés $^{(53)} »$. Ainsi, Stanley Fish, dans sa postface, n'hésite pas à écrire :

Par conséquent, si le texte lui-même n'est pas [...] une contrainte pour mon activité interprétative, les contraintes intériorisées de la communauté à l'intérieur de laquelle je travaille s'exercent puissamment (on pourrait même dire tyranniquement), et ce précisément parce que je n'en suis pas moi-même conscient ; elles sont la forme même de ma conscience ${ }^{(54)}$.

Il est vrai que Stanley Fish insiste sur le fait que ce ne sont pas les membres qui choisissent de rejoindre une communauté, mais que c'est la communauté qui les choisit « dans le sens où ses présupposés, préoccupations, distinctions, tâches, obstacles, récompenses, hiérarchies et protocoles deviennent, à la longue, l'aménagement même de leurs esprits, en les remplissant, selon la formule de l'ethnométhodologue Harvey Sacks "jusque dans les détails les plus minutieux"(55). . Bien sûr, il ne s'agit pas de remettre en cause le bien fondé et l'intérêt de ses analyses, mais force est de constater que Stanley Fish n'appréhende «sa propre question que d'un un seul point de vue, celui de la force contraignante des normes instituées et des systèmes d'interprétatifs intériorisés ", délaisse un autre point de vue beaucoup plus stimulant, celui du «mode de constitution de ces "communautés interprétatives" et [de] leur capacité à "fabriquer" le texte » :

Commencent s'agencent-elles? Quelle est leur écologie propre ? Comment se rapportent-elles à elles-mêmes : à leur mode de fonctionnement et de développement ? Comment se constituent-elles en tant que subjectivité collective ${ }^{(56)}$ ?

(51) Antoine Compagnon, Le Démon de la théorie, op. cit., p. 173.

(52) Pascal Nicolas-Le Strat, «Un usager faiseur de textes », art. cit., p. 133.

(53) Ibid.

(54) QLF., «Postface », p. 129.

(55) Ibid., p. 128.

(56) Pascal Nicolas-Le Strat, « Un usager faiseur de textes », art. cit., p. 134, pour 1'ensemble des citations. 
Le problème de l'émergence, de l'agencement et de la recomposition des communautés interprétatives reste donc ouvert. Une façon d'investir la question de la constitution de ces communautés et de leur écologie se trouve peut-être dans l'élargissement de la problématique vers des communautés d'usage, comme le suggère Pascal Nicolas-Le Strat. En effet, ce dernier considère que « le déplacement opéré par Stanley Fish [...] paraît essentiel car il resitue l'enjeu d'un texte dans un rapport d'usage et d' "utilité" et le réinscrit au sein d'une "communauté interprétative", en fait, sur un plan plus général, au sein d'une "communauté d'usage" (57). " Il serait donc urgent de démultiplier cette méthode qui nous renseigne sur ce que serait un «usager-faiseur de politique, faiseur de territoire, faiseur d'expertise ${ }^{(58)}$. » Car une politique des usages, selon Pascal NicolasLe Strat, a le mérite d'éviter deux écueils : " une conception toute puissance et fortement individualisée de l'usager (à l'image d'un lecteur désincarné, supposé complètement libre de ses interprétations) et l'idée d'un "usage" qui vaudrait pour lui-même et qui ferait loi en lui-même (à l'image d'un texte "fondamentalisé") ${ }^{(59)}$. » Il s' agit donc de «re-mettre au cœur de l'analyse (politique) la question des "communautés d'usage", à savoir les agencements collectifs susceptibles de constituer de nouveaux usages sur le mode d'une rappropriation créatrice, d'un détournement imprévisible et d'un piratage

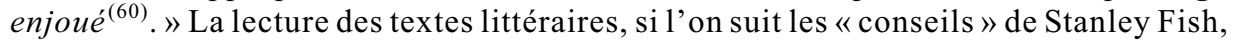
peut être considérée comme une propédeutique tout à fait intéressante à une telle redéfinition de la politique.

Mais avant tout, pour mon propos, il me paraît important de clarifier ce que Pascal Nicolas-Le Strat, en sociologue, entend précisément par « usage ${ }^{(61)} »-$ et c'est dans ce sens que j'emploierai ce terme dorénavant -, afin d'envisager quelques pistes qui permettraient de mieux cerner, autant qu'il est possible, ces « communautés d'usage » auxquelles appartiennent les lecteurs, et en particulier les faiseurs de textes. On notera que le terme d' " usage » est préféré à celui d' " usager », car Pascal Nicolas-Le Strat refuse « de rabattre l'usage sur la seule subjectivité de l'usager, et d'en faire en quelque sorte un simple dérivé(62) ». Il estime, en conséquence, qu'il est «possible de questionner la présence et l'agir d'un usage sans partir, pour autant, en quête des individualités qui seraient supposées l'avoir initié ou formulé. » Une telle hypothèse « invite à considérer l'usage comme un agencement, qui possède sa consistance propre, indépendamment des sujets qui l'impulsent, le traversent, le contredisent ». On peut donc supposer que tout usage dispose d'une « constitution ${ }^{(63)}$ » qui lui est spécifique. Il s'en suit que l'usage « fait "objectivement" trace », et ce sont ces traces (bribes de discours, comportements plus ou moins objectivés, fragments de réalité) qu'il faut saisir, et en particulier, en en tenant la chronique et en en faisant le récit, comme le fait Stanley Fish pour le sujet qui nous intéresse. On doit, néanmoins, garder à l'esprit que « les usages se formalisent imparfaitement; [qu' ils] ne sauraient se réduire à un mode d'emploi ${ }^{(64)}$ », car « la "constitution" d'un usage est [...] indissociable de la multiplicité de récits (sociologiques, photographiques, fictionnels, conversationnels...) auxquels il donne lieu ». Il s'a-

(57) Pascal Nicolas-Le Strat, «Un usager faiseur de textes », op. cit., p. 135.

(58) Ibid.

(59) Ibid.

(60) Ibid., p. 136 ; je souligne.

(61) Je reprends, dans le développement qui suit, les propositions de Pascal Nicolas-le Strat développées dans « Micropolitique des usages », in Moments de l'expérimentation, op. cit., pp. 19-29.

(62) Ibid., p. 19, et ce pour l'ensemble des citations qui suivent non référencées.

(63) Pascal Nicolas-Le Strat emploie ce mot dans le sens que lui a donné Michel Foucault, à savoir «comme l'entendraient les médecins, c'est-à-dire : rapport de force, équilibre et jeux de proportions, dissymétrie stable, inégalité congruente " (Michel Foucault, Il faut défendre la société (Cours au collège de France, 1976), Gallimard, 1997, p. 172).

(64) Pascal Nicolas-Le Strat, « Micropolitique des usages », art. cit., p. 20, et ce pour l'ensemble des citations qui suivent non référencées. 
girait donc, pour ce qui concerne l'interprétation des textes, de repérer les traces des usages dont la présence est attestée par leur «murmure insistant». En effet, un « usage fait nécessairement signe, le ferait-il sporadiquement ou par inadvertance». Ainsi pour rendre visibles et donner droit d'existence aux communautés d'usage - il faut insister sur la pluralité de celles-ci - auxquelles appartiennent les lecteurs dans leurs pratiques ordinaires et quotidiennes, il serait opportun « d'assumer une forme de "critique constituante" ", car :

Trop souvent, en effet, les sociologues - et en particulier les sociologues critiques - se comportent comme s'ils étaient des observateurs « réflexifs » et « distanciés » confrontés à des acteurs "naïfs", "non critiques » et non "réflexifs ». [...] Les acteurs sont tout à fait capables de proposer leurs propres théories de l'action afin d'expliquer comment les formes d'existence manifestent leurs effets ${ }^{(65)}$.

Ainsi, au lieu de s'évertuer à les cataloguer sur une échelle de légitimité (sociale, scolaire, "scientifique ») modélisée a priori, pourrait-on écouter et entendre, avec plus d'attention, les discours et les récits des lecteurs qui racontent comment ils s'emparent d'un texte et comment ils ont été saisis par lui, et prendre plus sérieusement en considération ce qu'on considère comme des mésinterprétations par rapport à l'étalonnage d'une lecture "normale », c'est-à-dire normée et normative qui a oublié la contingence de ses rites et de ses lois. Il faudrait repérer les usages mineurs que les lecteurs font des textes, en particulier ceux qui sont en deçà d'un seuil de visibilité, car trop singuliers et trop disruptifs par rapport aux modèles institutionnalisés dominants. Pour ce faire, il serait nécessaire, non seulement, déplier les usages pour en saisir les potentialités créatives jusque là refoulées, mais surtout les connecter à d'autres phénomènes liés au contexte dans lequel ils émergent. Car un usage, transgressif, insère ses propres agencements dans les pratiques dans lesquelles il se manifeste : " Il s'intercale et dérègle leur fonctionnement. Il leur accorde de la sorte un surcroît d'existence, inattendu, important, parfois inespéré. Un usage insuffle de nouvelles intensités de vie : de l'étonnement et de l'agacement, des tensions et du plaisir ${ }^{(66)}$. » Ce n'est qu'à cette condition que l'on pourra accorder à un usage indiscipliné un corps, c'est-à-dire un supplément de réalité. Une telle approche « constituante » permettrait de retrouver la «constitution » d'un usage, c'est-à-dire quelque chose qui a «consistance et situation historique ${ }^{(67)}$ » et qui s'incarne dans des réalités matérielles ou imaginaires, relationnelles ou spatiales, intellectuelles ou affectives.

Une des caractéristiques essentielles des usages, fait remarquer Pascal Nicolas-Le Strat, est leur «polyvalence tactique ${ }^{(68)}$ », car, équivoques et réversibles, ils peuvent, tout à la fois, concrétiser les habitudes établies, c'est-à-dire « œuvrer à la reconduction fonctionnelle de l'existant ${ }^{(69)}$ » et faire événement, en transgressant les « fonctionnalités admises ou acquises ». Il n'est donc pas étonnant que les institutions recourent à des " trésors d'ingéniosité » pour endiguer la survenue d'usages non conformes. Il faut avouer que, par exemple, l'école apparaît comme une machine redoutable pour récupérer, normaliser et standardiser des usages d'interprétation et de lecture des textes, qui,

(65) Bruno Latour, Changer de société - Refaire la sociologie, La Découverte, 2006, pp. 82-83 ; cité par Pascal Nicolas-Le Strat, « Micropolitique des usages », art. cit., pp. 26-27.

(66) Ibid., p. 25 ; je reviendrai sur l'étonnement, l'agacement et le plaisir que procurent les affabulations théoriques de Stanley Fish.

(67) Michel Foucault, op. cit.

(68) Pascal Nicolas-Le Strat transpose sur le terrain des usages la formulation de Michel Foucault « La polyvalence tactique des discours » (in Histoire de la sexualité 1 - La volonté de savoir, Gallimard, 1976, p. 133) ; je souligne.

(69) Pascal Nicolas-Le Strat, « Micropolitique des usages », art. cit., p. 22-23, et ce pour 1'ensemble des citations qui suivent. 
lors de leur émergence, voulaient rompre avec les modèles hégémoniques en cours ${ }^{(70)}$. Et se pose donc, expressément, la question de savoir comment se défaire de l'emprise des usages les plus normés socialement, comment « amorcer une autre perspective, opérer les torsions et les détournements indispensables ». Il est incontestable que les thèses de Stanley Fish permettent de penser comment l'interprétation des textes peut devenir un « art indiscipliné », difficilement « récupérable ou instrumentalisable». Des théoriciens de la littérature comme Marc Escola ou Pierre Bayard ont ouvert des voies exemplaires à cet égard ${ }^{(71)}$.

Mais « un usage ne s'impose jamais d'un seul élan; il chemine, il progresse à travers les pesanteurs du quotidien et se heurte fréquemment aux normes de fonctionnement », car c'est sa « banalité insistante qui parvient à saper l'ordonnancement habituel ${ }^{(72)}$ " des pratiques. Un usage, surtout s'il est en rupture avec les codes en vigueur, n'a de chance de se pérenniser et de confirmer sa validité que s'il revient vers la vie quotidienne. Car «l'usage accède à une quotidienneté ou se disperse, s'étiole, s'altère. C'est à l'épreuve du quotidien qu'il affermit son autonomie. C'est par l'entremise de minuscules initiatives, récurrentes, répétitives, qu'il manifeste une liberté. » Un usage devient scandaleux et indispose précisément au moment où il fait entendre " l'insistance humble et murmurante » de sa "présence opiniâtre». On le voit toute théorie littéraire - et a fortiori toute pédagogie de la lecture - qui voudrait rompre avec des usages qu'elle jugerait peu satisfaisants se trouve dans l'obligation de résoudre une équation dont les tentatives de réponse peuvent apparaître aporétiques : Comment ne pas se faire récupérer et assimiler par les systèmes institutionnalisés ? Comment occuper le quotidien ? On peut le faire, peut-être en créant des communautés d'usage autour d'expérimentations, car expérimenter «c'est constituer un contre-pouvoir à l'intérieur même des situations [...], c'est faire advenir de nouvelles formes de vie et d'activité, de pensée et de création [...], c'est opposer aux dispositifs de domination une puissance d'autonomie et de singularisation [...], c'est déployer une question à l'endroit même où les institutions imposent une solution ${ }^{(73)}{ }^{\prime}$.

Selon Pascal Nicolas-Le Strat, un usage est donc :

[...] un agencement hétérogène dont il est difficile de poser a priori les limites. Il ne se définit pas en soi, comme tel. Il n'incorpore pas en lui une logique irréductible qui s'appliquerait dans n'importe quel contexte et qui se déclinerait, égale à elle-même, en toute situation. Il n'inclut pas son mode d'emploi. Un usage représente avant tout une façon de se rapporter à soi et aux autres, à soi et à son contexte de vie. Il est sans cesse relancé et stimulé par des interactions auxquelles il s'adapte ou auxquelles il s'oppose. Il est en permanence branché sur une multiplicité d'extériorités qui le confrontent à ses ressources et à ses potentialités ${ }^{(74)}$.

Pour en rester à l'interprétation des textes, il serait donc opportun, comme nous l'y invite Pascal Nicolas-Le Strat, de voir dans quelle mesure les hypothèses développées par Stanley Fish, sont capables de moduler, de déborder et de miner de l'intérieur la doxa qui régule l'interprétation des textes tant dans les institutions que dans la vie ordinaire. Il n'en reste pas moins qu'il faut, aussi, s'employer à mettre à jour les résistances

(70) Le sort qui a été fait, par exemple, à la narratologie ou à la poétique du récit est exemplaire à cet égard.

(71) Voir infra.

(72) Pascal Nicolas-Le Strat emprunte cette formulation à Judith Butler (Humain, inhumain (Le travail critique des normes) Amsterdam, 2005, p. 57) ; cette citation et celles qui suivent renvoient aux pages 24-25 de «Micropolitique des usages », art. cit. ; je souligne.

(73) Pascal Nicolas-Le Strat, «Introduction », Moments de l'interprétation, op. cit., p. 11 ; je souligne.

(74) Pascal Nicolas-Le Strat, « Micropolitique des usages », art. cit., p. 28. 
que pourrait rencontrer un tel changement de paradigme dans l'interprétation des textes, afin de ne pas s'immobiliser, quand les pressions s'exacerbent, « dans un vis-à-vis stérile et inhibant, sans parvenir à amorcer de lignes de fuite ou de contournement ${ }^{(75)} »$. Car un usage, par définition, est connecté « tant en extériorité qu'en intériorité, à d'innombrables "entités", qu'elles soient matérielles ou symboliques, relationnelles ou fonctionnelles ${ }^{(76)} »$; ou, dit autrement, un usage est obligé de s'ajuster à la fois « aux nombreuses extériorités qui le mettent à l'épreuve et l'obligent [...] et à ses intériorités, par rapport à la multiplicité qui se déploie en lui : des désirs, des habitudes, des objets familiers, des rituels, des schèmes d'action ${ }^{(77)} »$. On comprend donc pourquoi Pascal Nicolas-Le Strat attribue un caractère profondémentécosophique ${ }^{(78)}$ à tout usage, puisqu'il est « avant tout une modalité d'entrer-en-rapport, de rejouer son rapport à soi ou à son environnement ${ }^{(79)}$. »

Yves Citton ${ }^{(80)}$, dans le commentaire qu'il fait de l'expression employée par Stanley Fish pour décrire ce que font les interprètes - They make them-, insiste, lui aussi, sur la nécessité de raisonner en terme d'usage. En effet, dans une première acceptation, to make signifie que «ce sont les interprètes quifabriquent les poèmes, qui les produisent, qui les créent, qui les font émerger en appliquant sur un texte certaines recettes de cuisine (intériorisées au fil des semaines selon le formatage mental agencé par le professeur Fish). Le modèle sous-jacent à premier sens du verbe to make est celui du Créateur (Maker) qui donne être à quelque chose qui n'existait pas avant lui. » C'est bien sûr une telle compréhension de ce verbe qui a pu alimenter les critiques ironiques qui ont accablé Stanley Fish, en mettant en exergue l'absurdité d'une telle proposition : " de même que c'est évidemment la boulanger qui produit la pain (et non le client, qui l'achète et le mange), de même est-ce évidemment le poète qui produit le poème (et non le lecteur, qui le déchiffre). » Mais, précise Yves Citton, il existe un second sens de to make qui correspond mieux à ce qu'ont fait les étudiants de Stanley Fish avec la liste qui leur était proposée :

L'anglais emploie le même verbe to make pour dire qu'une mauvaise nouvelle me rend triste ou que des cacahouètes trop salées me rendent assoiffé (it makes me sad, it makes me thirsty). Pour être plus littéral, et plus littéraire, il ne faudrait pas dire que les interprètes «font» les textes, mais que, en présence d'un texte déjà fait, les interprètes peuvent en faire un poème. Il ne s'agit pas de créer, mais d'utiliser, et, du point de vue pragmatiste, il n'est aucune utilisation « illégitime » d'un objet, dès lors qu'elle remplit le but visé.

Ainsi, on l'a vu, les étudiants de Stanley Fish, qui appartenaient à deux communautés interprétatives différentes, ont fait un usage différent des mêmes traces écrites, pour les uns une liste de livres à lire, pour les autres un poème religieux. Pour un pragmatiste, rappelle Yves Citton, aucun des deux usages ne peut être dit plus adapté à une " essence » de l'objet précédant ces usages : les objets ne sont que ce que nous en faisons. Certes, il reste la question de la sous-utilisation d'un objet, par exemple caler un meuble avec un recueil de poèmes, obstruer une fenêtre avec un tableau, allumer un barbecue avec un billet de banque... Cette sous-utilisation n'est pas à interpréter comme un

(75) Ibid., pp. 28-29.

(76) Ibid.

(77) Ibid., p. 29.

(78) Cf. Félix Guattari, Les trois écologies (Paris, Galilée, 1989) ; l'idée d'écosophie articule les trois écologies : environnementale, sociale et mentale.

(79) Pascal Nicolas-Le Strat, « Micropolitique des usages », art. cit., p. 29 ; je souligne.

(80) QLF., Yves Citton, «Puissance des communautés interprétatives », pp. 21-22, pour tout le développement et les citations qui suivent. 
manque de « respect» avec l'objet utilisé, mais manifeste deux faiblesses : d'une part, elle n'est pas conforme aux habitus en vigueur dans les communautés d'usage, et d'autre part elle produit « un manque à gagner pour l'utilisateur». On conviendra aisément que, dans des circonstances habituelles, il est plus avantageux et plus fructueux d'installer sur sa fenêtre une vitre, et de lire un livre pour se détendre ou pour vivre une expérience esthétique. Telles sont, selon William James, la méthode et l'attitude pragmatistes qui consistent précisément « à détourner son regard des causes premières, des principes et des catégories dans lesquelles on voit des nécessités, pour se tourner plutôt vers les fruits, les conséquences et les faits », car les «idées (qui ne sont rien d'autre que des parties de notre expérience) deviennent vraies dans la stricte mesure où elles nous permettent d'entrer dans des relations satisfaisantes avec d'autres parties de notre expérience $^{(81)}$.» Il est aisé d'appliquer à l'art, à la littérature, ce qui est dit ici de la vérité. Il n'en reste pas moins que ces détournements des usages est toujours possible, comme nous le montre à l'envi l'art contemporain, pratiquant avec délices la « transfiguration du banal ${ }^{(82)} »$.

\section{Un parti pris pragmatiste}

La réflexion de Stanley Fish ne relève pas seulement d'un tempérament joyeusement - certains esprits chagrins diront tristement - iconoclastique. Elle s'inscrit dans un courant philosophique, le pragmatisme, qui lui confère une portée qu'on ne peut négliger dans la réflexion théorique qui se développe dans le champ littéraire, et en particulier sur des notions qui nous préoccupent ici : la littérarité, l'usage et le contexte. Richard Rorty ${ }^{(83)}$ en est un des représentants les plus éminents ; et il me semble qu'on peut trouver chez ce philosophe des échos, des approfondissements et des prolongements tout à fait passionnants des positions défendues par Stanley Fish.

Richard Rorty est, lui aussi, un antifondamentaliste rigoureux et conséquent pour qui tout point de vue absolu sur la réalité est impossible. Il rejette l'idée, mythique et religieuse ${ }^{(84)}$, d'une vérité transcendante - quel que soit le nom qu'on lui donne : les « faits », l' « objectivité », le « réel »-qui serait propre à fonder nos croyances et nos représentations. Il faut, au contraire, considérer que notre rapport au monde ne peut être que de nature causale et non représentationnelle, puisqu'il est impossible de justifier, en dernière instance, l'adéquation de nos croyances à une réalité qui leur préexisterait. Cette position amène Richard Rorty à opposer deux figures de penseurs : le métaphysicien essentialiste et l'ironiste anti-essentialiste ${ }^{(85)}$, vers qui va sa préférence :

(81) William James, Pragmatism, Indiapolis, Hackett Publishing Co., 1991 ; cité par Jean-Pierre Cometti, Art, représentation, expression, Paris, PUF, coll. "Philosophies », 2002, p. 118 ; je souligne.

(82) Cf. Arthur Danto, La Transfiguration du banal. Une philosophie de l'art, 1981, trad. de l'anglais (américain) par C. Hary-Schaeffer, préface de J.-M. Schaeffer, Paris, Éd. du Seuil, coll. « Poétique », 1989.

(83) Il ne s'agit pas ici de « résumer» la philosophie de Richard Rorty en quelques phrases, ce qui serait vain, et à coup sûr ridicule, mais de signaler seulement quelques points de convergence entre le philosophe et le théoricien de la littérature, Stanley Fish.

(84) "Si la vérité n'est pas un vain nom, elle n'est dans l'univers qu'à l'état d'expression, et dans notre esprit qu'à l'état d'apparition; elle est dans l'univers comme l'artiste dans son œuvre, elle est dans notre esprit comme le soleil dans nos yeux. Mais par delà l'univers et notre esprit, elle subsiste en ellemême, elle est une essence réelle, infinie, éternelle, absolue, existant par soi, ayant conscience et intelligence de soi ; car, comment la vérité ne s'entendrait-elle pas elle-même, puisqu'elle est la source de tout entendement? Or, dire cela de la vérité, c'est définirDieu; Dieu est le nom propre de la vérité, comme la vérité est le nom abstrait de Dieu. » H.-D. Lacordaire, Conférences de Notre-Dame, 1848, p. $26-T L F$, article « absolu»; je souligne.

(85) Je reprends ici le développement de Richard Rorty, Contingence, ironie \& solidarité, 1988, trad. de l'anglais (américain) par P.-E. Dauzat, Paris, A. Colin, coll. «Théories », 1993, pp. 117-118, pour l'ensemble des citations non référencées qui suivent; je souligne. 
Le philosophe essentialiste, celui qui entend bien s'accrocher à l'idée de "propriété intrinsèque ", indépendante de tout contexte, déclare pour sa part que la recherche contextualiste doit être quelque chose de précontextuel. À quoi l'anti-essentialiste réplique en faisant valoir qu'aussi loin que l'on aille, il n'y a jamais que des contextes. Car pour lui, notre recherche ne peut porter que sur des choses associées à une description ; la description d'une chose consiste à établir une relation entre elle et d'autres choses, et la possibilité de "saisir la chose elle-même" ne précède pas la contextualisation ${ }^{(86)}$.

Le métaphysicien essentialiste considère donc que les différentes théories philosophiques sont convergentes dans la mesure où elles présentent des arguments à l'appui de leurs points de vue, pour se rapprocher de plus en plus de la nature des choses, de la réalité, et en dernière instance de la vérité. En revanche, pour l'ironiste ${ }^{(87)}$ anti-essentialiste, la succession des théories réside, en fait, dans « des substitutions progressives et tacites d'un vocabulaire nouveau à un vocabulaire ancien », afin que les gens changent de pratique, en adoptant un «nouveau jargon », sans admettre toujours qu'ils l'ont fait. Et, donc, « la forme de raisonnement qui a les préférences de l'ironiste est dialectique en ce sens que, pour lui, l'unité de persuasion est un vocabulaire plutôt qu'une proposition. » Pour cela, il faut adopter une méthode dialectique, c'est-à-dire substituer partiellement la (re)description à l'inférence pour faire « jouer les vocabulaires les uns contre les autres, plutôt que de simplement inférer des propositions les unes des autres ». Telle est la démarche qu' ont suivie des philosophes comme Nietzsche, Heidegger et Derrida, qui « définissent leur œuvre par rapport à celle de leurs précédents plutôt que par rapport à la vérité ». La méthode dialectique n'est donc en rien une procédure d'argumentation, mais procède d'un réel «talent littéraire », et ajoute Richard Rorty, "pour désigner ce que j'ai nommé "dialectique", il y aurait une expression plus moderne : celle de "critique littéraire" ". On ne saurait mieux dire...

Stanley Fish et Richard Rorty se rejoignent donc pour affirmer que les objets ne sont jamais donnés, innocemment, dans une autonomie absolue, car ils n'existent que dans une « sémiosphère ${ }^{(88)}$ ", c'est-à-dire dans des discours divers - qu'ils se présentent et soient reçus comme institutionnels ou libres, comme centraux ou périphériques, comme scientifiques ou doxiques. On comprend, dès lors, pourquoi les concepts de vocabulaire, de (re)description et de (re)contextualisation sont au cœur de la réflexion menée par Richard Rorty. En effet, tout essentialisme menant, inévitablement, à une impasse et à des apories insurmontables, nous n'avons que la possibilité de redécrire les différents éléments qui composent nos croyances et qui font nos mondes. Et, pour ce faire, le meilleur moyen est de les recontextualiser, c'est-à-dire de les saisir, délibérément, dans un nouveau contexte. Si nous nous en tenons à la critique littéraire, nous voyons que comprendre et interpréter un texte ne peut consister à construire, au préalable, un artefact illusoire dont on stipulerait la pureté scientifique et objectale (son contexte d'origine, sa structure linguistique et/ou sémiotique, etc.), par un geste qui annulerait ou suspendrait le contexte et la situation du lecteur critique faiseur de textes. Il faut, au contraire, reconnaître que, tout texte étant solidaire d'un contexte, nous n'a-

(86) Richard Rorty, Objectivisme, relativisme et vérité, op. cit., p. 115.

(87) Richard Rorty, Contingence, ironie \& solidarité, op. cit., pp. 111-112 : «L'ironiste, suivant ma définition, est quelqu'un qui remplit trois conditions : (1) il a des doutes radicaux et permanents sur le vocabulaire qu'il emploie couramment, parce que d'autres vocabulaires lui ont fait forte impression, des vocabulaires pris comme finaux par les gens ou dans les livres qu'il a rencontrés ; (2) il réalise que le raisonnement formulé dans son vocabulaire présent ne peut ni confirmer ni dissoudre ces doutes ; (3) pour autant qu'il philosophe sur sa situation, il ne pense pas que son vocabulaire soit plus proche de la réalité que les autres [...]. [Pour lui] le choix entre les vocabulaires ne se fait pas au sein d'un métalangage neutre et universel, ni en essayant de se frayer un chemin vers le réel à travers les apparences, mais simplement en jouant le nouveau contre l'ancien. »

(88) J'emploie ici le vocabulaire de Youri Lotman, La Sémiosphère, 1966, trad. du russe par A. Ledenko, Limoges, PULIM, coll. « Nouveaux Actes Sémiotiques », 1999. 
vons affaire qu'à du déjà-dit, qu'à du déjà-écrit. Il s'en suit que la redescription d'un texte va consister précisément à faire varier le(s) contexte(s) de son interprétation. Pour Richard Rorty - et la « leçon » de Stanley Fish mène à la même conclusion -, interpréter c'est faire dialoguer un contexte d'intelligibilité avec/contre un autre. On peut, par exemple, jouer sur la diachronie - le nouveau contre l'ancien, en quelque sorte -, sur l'interdisciplinarité ou sur l'indisciplinarité ${ }^{(89)}$, chère à Stanley Fish qui s'est efforcé, quant à lui, de lire des textes de droit en fonction de réquisits littéraires, ce qui n'est pas sans conséquences, en retour, sur l'idée que l'on peut se faire de la littérature et de son usage, car, comme l'indique Yves Citton dans sa préface, «[...] il s'agit de creuser (disciplinairement) sa discipline jusqu' au point où 1 'on met à nu son manque de fondations, et où ce trou nous ouvre un souterrain vers le manque de fondations d'une autre discipline. De ce dialogue entre droit et littérature, les disciplines ne sortent pas enrichies ni supplémentées l'une de l'autre, mais sapées, et sommées de se reconstituer sur des bases nouvelles et déniaisées ${ }^{(90)}$. »

Autrement dit, toute redescription prend la forme d'une recontextualisation, dont l'objectif est de connecter l'objet redécrit avec une nouvelle théorie explicative ${ }^{(91)}$, une nouvelle classe de comparaison, un nouveau vocabulaire descriptif, un nouveau livre qu'on a lu, une expérience inédite qu'on a vécue. L'activité critique consiste donc à redécrire un texte, afin de lui donner une nouvelle valeur d'usage, et ce en le connectant à des jeux de langage connectés à ces mêmes usages.

Ce parti pris d'une esthétique pragmatiste est défendu, aussi, par Jean-Pierre Cometti. Ce philosophe a, pour moi, le mérite de mettre au centre de ses réflexions la notion d'usage et de permettre, ainsi, d'élargir l'horizon des conceptions défendues par Stanley Fish, en empêchant qu'on les restreigne à une énième version d'une théorie sociologique de l'art, comme pourrait le suggérer malencontreusement la notion de « communauté interprétative ». Tout d'abord, d'une façon, à première vue, provocatrice, il affirme que l'art, et donc la littérature, est « sans qualités ${ }^{(92)} »$ :

Telles sont les raisons pour lesquelles j'ai choisi de parler d' « Art sans qualités » [...] ; non pas, comme on aurait tort de le croire, pour exprimer je ne sais quel jugement de valeur : 1'absence de qualités n'a rien à voir ici avec une absence de prix, sinon en cela que l'art ne possède en lui-même, de manière intrinsèque, aucun prix, pas plus qu'aucun attribut, aucune propriété, fût-elle séparable de nos évaluations, que nous aurions quelque chance de discerner et de prédiquer en connaissance de cause. Autrement dit, ce que marque l'absence de qualités, dans ce cas précis, est à peu près l'équivalent de ce que Musil appelait l'amorphisme humain, la vanité d'une essence présumée, quelque chose comme une ontologie neutre $[\ldots]^{(93)}$.

(89) "Indisciplinarité : attitude de recherche et de réflexion cherchant non seulement à croiser horizontalement les approches développées par différentes disciplines (comme le fait l'interdisciplinarité), mais aussi à intégrer verticalement les sensibilités et les savoirs développés par chaque individu au sein des différentes sphères de son existence (professionnelle, artistique, citoyenne, religieuse, sportive, etc.) »-Yves Citton, Lire, interpréter, actualiser. Pourquoi les études littéraires?, Paris, Éd. Amsterdam, 2007, p. 343.

(90) $Q L F$., Yves Citton, «Puissance des communautés interprétatives », p. 12.

(91) C'est ce que fait, exemplairement, Roland Barthes dans son Sur Racine (Paris, Éd. du Seuil, coll. "Points Essais», 1979) en confrontant le texte racinien au discours psychanalytique, et en particulier au « roman» freudien de la « horde sauvage». Son détracteur, Raymond Picard, est le prototype d'un critique essentialiste.

(92) Jean-Pierre Cometti, dans L'Art sans qualités (Tours, Farrago, 1999), propose une redéfinition de l'art à partir de la notion de l'Eigenschafstlosigkeit (mot qui signifie l'absence de qualités propres) empruntée à Musil (Robert Musil, L'Homme sans qualités, trad. de l'allemand par Philippe Jaccottet, Paris, Éd. du Seuil, 1957).

(93) Ibid., p. 11 ; je me réfère à l'édition mise en ligne ; consultée le 2 août 2011 ; URL http://www.cheminderonde.net/documents/1.pdf ?PHPSESSID=4098231b4800 dc67512e58af50fcec8d. 
Pour Jean-Pierre Cometti, comme pour Richard Rorty et Stanley Fish, 1'art - tout objet en fait - est sans attributs ou propriétés essentielles, qui pourraient réellement être tenus pour propres, car on ne saurait isoler des propriétés intrinsèques des œuvres d'art hors du contexte qui les reconnaît et les fait fonctionner comme telles ${ }^{(94)}$. Toutefois, la prise en compte des modalités institutionnelles, dans l'activation des œuvres d'art, ne conduit pas forcément le philosophe à souscrire, sans recul, aux théories institutionnelles de l'art ${ }^{(95)}$. En effet, une telle conception, outre son caractère étroitement nominaliste, circulaire sinon tautologique - «l'art, c'est l'art »-, reconduit la dichotomie, qu'elle est censée rejeter, entre les pratiques artistiques, confinées au monde de l'art, et l'ensemble de la société. Ce qui l'amène à ne pas prendre en compte les usages très divers et irréductibles au monde de l'art qui sont faits des œuvres. En effet, l'absence de qualités implique que toute activation d'une œuvre tisse un réseau de relations avec les autres jeux de langage, artistiques ou non, qui sont forcément contingents, variables et divers. L'œuvre apparaît donc toujours dans des contextes pluriels, " sans que la communication en soit réglée a priori, et sans qu'une syntaxe y soit tenue pour souveraine $^{(96)} »$. On peut parler de véritable paradoxe de l'usage, comme le fait Jean-Pierre Cometti :

Que l'art soit « sans qualités » au sens où cette notion a été mobilisée jusqu'ici, cela ne veut pas seulement dire que la possibilité en excède toujours quelque essence ou quelque origine que ce soit; plus profondément, peut-être, «l'usage»-en entendant par là ce qui ne saurait être détaché ni pensé indépendamment de ce que nous faisons - me semble en être la « règle », l'erreur majeure consistant précisément à imaginer une règle antérieure à toutes ses applications, et qui en serait ainsi indépendante ${ }^{(97)}$.

La perception et la compréhension d'une œuvre sont fonction d'une relation, dans un certain contexte, entre l'œuvre comme telle et un lecteur; il faut entendre par là pour Jean-Pierre Cometti que «l'œuvre et [le lecteur] nouent une relation qui n'est pas seulement définissable dans un contexte déterminé, mais selon un ensemble de dispositions égalementdéterminées ${ }^{(98)}$. \Car cette relation est toujours connectée certes à une situation, à un contexte de pratiques données, mais aussi à une forme de vie, pour reprendre l'expression de Wittgenstein ${ }^{(99)}$, c'est-à-dire à l'ensemble de nos jeux de langage. «Autrement dit, il y a toujours à l'arrière-plan, un contexte de pratiques communes, un langage, qu'implique la nature sémantique des phénomènes concernés ${ }^{(100)}$. » C'est pourquoi la première tâche, selon John Dewey, qui incombe à celui qui entreprend de réfléchir sur l'art, sur la littérature a fortiori, est de rétablir le lien qui l'unit aux autres dimensions de l'expérience, en restaurant « la continuité entre les formes d'expérience épurées et renforcées que sont les œuvres d'art et les événements, les actions et les souffrances du quotidien qui sont universellement reconnues comme constituant l'expé-

(94) La position de Jean-Pierre Cometti est tout à fait intéressante et originale, car elle évite à la fois les apories de l'essentialisme esthétique ( «les œuvres d'art se définissent comme telles par des propriétés qu'elles possèdent de manière intrinsèque et constitutive, indépendamment des relations que nous entretenons avec elles ») et du subjectivise ou du relativisme ( les propriétés que nous attribuons aux œuvres d'art sont de nature relationnelle et ne doivent rien à ce qu'elles possèdent en propre »). Ibid., p. 63.

(95) Voir en particulier les analyses d'Arthur Danto et de Georges Dickie, « Définir l'art », in G. Genette (éd), Esthétique et poétique, Paris, Le Seuil, 1992. L'art pour ces auteurs est défini comme ce qui est homologué comme tel par le "monde de l'art».

(96) Jean-Pierre Cometti, L'Art sans qualités, op. cit., p. 48.

(97) Ibid., p. 52.

(98) Jean-Pierre Cometti, Art, représentation, expression, op. cit., p. 74 ; souligné par 1'auteur.

(99) Voir Jean-Pierre Cometti, Philosopher avec Wittgenstein, $2^{\circ}$ édition, Paris, Farrago, 2001, chap. 4 et 5 .

(100) Jean-Pierre Cometti, Art, représentation, expression, op. cit., p. 59 ; je souligne. 
rience $^{(101)}$. » Le fait de considérer l'art comme une expérience, note Jean-Pierre Cometti à propos des thèses de John Dewey, permet de «mettre en relief les conditions opératoires, fonctionnelles et contextuelles qui, à la différence des théories qui privilégient l'objet, montrent toute l'importance de ce que l'art fait ${ }^{(102)}$. "

Dire que «l'art est sans qualités » ne veut pas dire, évidemment, que l'on ne peut attribuer à un objet des propriétés ; il s'agit seulement de «faire valoir que ces propriétés ne possèdent pas un caractère esthétique et/ou artistique à proprement parler (telles qu'elles assureraient aux objets le statut ontologique d'œuvres d'art), et que l'esthétique est affaire de fonctionnement, lié à des conditions elles-mêmes variables ${ }^{(103)}$. » Il n'existe que des «symptômes de littérarité », et pas de caractères ou d'attributs en euxmêmes déterminants la nature littéraire d'un objet textuel. La question qui nous préoccupe $^{(104)}$ n'est donc pas «Qu'est-ce que la littérature ?», mais bien « Quandy a-t-il littérature?»

Ainsi, est sans objet et caduque la question - malicieuse, sinon ironique - de savoir si la critique aurait encore une raison d'être pour une théorie qui soutiendrait qu'il n'existe pas, dans les textes, de qualités intrinsèques autonomes. L'existence de la critique n'est pas remise en cause, même si elle n'est plus confrontée à la tâche impossible de devoir rendre compte « objectivement» de qualités essentielles. En effet, souscrire à une telle ontologie «neutre » de l'œuvre, comme nous y invite Jean-Pierre Cometti, ne signifie pas qu' on déclare que le texte est « vide», mais, bien au contraire, c'est assumer qu'il est fait de contextes, à savoir ceux que les usages qui se sont emparés de lui ont fait apparaître, ou, autrement dit, qu'il est constitué des anciennes descriptions dont il a été l'objet, descriptions elles-mêmes connectées à d'anciens usages. Pour reprendre le vocabulaire de Richard Rorty, ont peut dire que la critique littéraire, parce qu'elle est une opération recontextualisante, engendre des redescriptions qui connectent les œuvres d'art à des usages divers - puisque ce sont les usages qui fixent et définissent les «propriétés » des objets artistiques. Ainsi, elle peut proposer des contextes d'intelligibilité nouveaux et se faire confronter des épistémès qui jusqu'alors s'ignoraient. En effet, affirme Jean-Pierre Cometti, en suivant en cela les indications de Wittgenstein, «nos jeux de langage esthétiques communiquent, en un sens, avec la totalité de nous jeux de langage, raison pour laquelle l'art est toujours hors de soi. [Ainsi] en décrivant de manière pragmatique l'art comme un jeu de langage non isolable, connecté à d'autre jeux de langage, et inscrit dans des formes de vie, on se donnerait les moyens de glisser simplement dans les usages de l'art pour mieux le(s) saisir. ${ }^{(105)} »$. Il s'agit donc, d'une certaine façon, d'une part de refuser l'isolement des études littéraires - effet induit par la proclamation performative de l'autonomie et de l'autotélicité de la littérature par la doxa romantique -, et de réarticuler les différentes disciplines entre elles, en mettant en œuvre une véritable interdisciplinarité, c'est-à-dire un dialogue entre des disciplines comme l'histoire, la sociologie, l'anthropologie, la philosophie, la critique littéraire.... Et ce, tout en n'oubliant pas que la littérature, comme art, demeure un opérateur précieux d'indisciplinarité, comme on l'a vu, car, précise Jean-Pierre Cometti, les usages que nous en faisons nous forcent et nous apprennent à faire bouger les lignes, les citadelles disciplinaires et tout particulièrement les linéaments de nos formes de vie.

C'est donc la question du contexte qui détermine la pertinence d'une interprétation,

(101)John Dewey, Art as Experience, 1934, A. Perigee Book, 1980 ; cité par J.-P. Cometti, Art, représentation, expression, op. cit., p. 115 ; je souligne.

(102)Ibid., p.. 116-117; souligné par l'auteur.

(103) Jean-Pierre Cometti, L'Art sans qualités, p. 52 ; je souligne.

(104)Cf. Nelson Goodman, "Quand y a-t-il de 1'art", in Manières de faire des mondes, 1978, trad. de 1'anglais (américain) par M.-C. Popelard, Paris, J. Chambon, 1992, pp. 89-90.

(105)Jean-Pierre Cometti, L'Art sans qualités, op. cit., p. 72 ; je souligne. 
et non une hypothétique fidélité à l'objet qu' elle décrit. Cependant, il ne s'agit pas d'accepter telles quelles toutes les redescriptions ; à l'évidence certaines sont peu économiques - pas en coût herméneutique, mais en coût pratique - lorsqu'elles s'écartent trop nettement des usages communément pratiqués dans une communauté interprétative, lorsque leur pouvoir connectif à d'autres discours et à d'autres redescriptions est trop faible, et lorsqu' elles n' augmentent en rien notre pouvoir de connaissance et notre puissance d'exister.

\section{La portée subversive de l'œuvre de Stanley Fish}

Dans sa préface, Yves Citton montre, très justement, combien l'œuvre de Stanley Fish, une bombe à retardement, a une portée subversive :

S'il faut lire Fish aujourd' hui, c'est au titre d'une bombe à retardement. Vingt cinq ans après leur parution dans leur langue originale, les essais consacrés à la dimension projective de l'interprétation non seulement gardent toute leur pertinence, mais s'avèrent tirer un sens et une urgence renouvelés à la lumière des évolutions récentes des débats publics et des mentalités ${ }^{(106)}$.

En effet, rappelle-t-il, la théorie du lecteur-faiseur de textes a vu le jour, aux ÉtatsUnis, dans le contexte, « de la vague émancipatrice et créatrice qui avait culminé avec les mouvements sociaux de la fin des années 1960 et du début des années $1970^{(107)} »$. Il est difficile d'ignorer combien cette thèse est d'actualité, aujourd'hui, dans un contexte de retour à tous les fondamentalismes, qu'ils soient politiques, religieux ou économiques. Et l'on peut penser, comme Yves Citton, que les essais de Fish peuvent devenir un outil de résistance, une machine de guerre ${ }^{(108)}$ et une aide précieuse pour « défondamentaliser » les situations et les croyances, et pour « ouvrir des possibles là où nous croyions faussement que la nature ou un Texte sacré nous imposent une solution unique - une seule "bonne" interprétation, inhérente à la chose elle-même ${ }^{(109)}$. » C'est donc une tâche politique de salut publique que « de nous convaincre qu'aucun texte ne prescrit quoi que ce soit par lui-même, mais que ce sont toujours les interprètes (humains) qui font dire à ce texte quelque chose qui leur est utile ${ }^{(110)}$. » Car il ne fait pas de doute, pour Yves Citton, que la théorie du lecteur-faiseur de texte «peut être mise en série avec d'autres formes contemporaines de "libération", d'encapacitation (empowerment) et de revendication d'“autonomie" (111). " S'il y a une leçon politique à tirer des fables théoriques de Stanley Fish, ce n'est pas, comme on l'a vu, tant celle d'une liberté interprétative débridée, que celle d'une «reconnaissance du caractère conditionné de toute interprétation ${ }^{(112)}$. " La notion même de communauté interprétative signale qu'une interprétation ne relève pas des catégories du «vrai» ou du « faux », mais de l'acceptable ou de l'inacceptable, eu égard à un contexte d'énonciation et de réception. Ce sont précisément les communautés interprétatives «qui donnent aux subjectivités individuelles leurs formes, leurs limites et leurs visées ${ }^{(113)}$. »

Une telle conception implique, par ailleurs, au moins deux conséquences, dont la portée politique, là aussi, n'est pas à négliger. D'une part, sont remises en cause, dans

(106)QLF., p. 17 ; souligné par l'auteur.

(107)Ibid., Yves Citton, «Préface », p. 17.

(108)Ces expressions sont d'Yves Citton, Ibid., p. 26.

(109)Ibid.

(110)Ibid., p. 25 ; souligné par l'auteur.

(111) Ibid.

(112)Ibid., p. 18 ; souligné par l'auteur.

(113)Ibid., p. 20. 
les études littéraires, l'hégémonie et l'arrogance des approches scientistes - les «sciences du texte » n'en sont pas dépourvues - qui tentent de définir et de fonder la littérarité d'un texte à partir de propriétés formelles. La détermination de la littérarité des textes, comme on l'a vu, ne dépend pas de la possession de traits littéraires essentiels et inhérents, mais de l'usage qui est fait de ces textes, dans un certain contexte, usage réglé par une communauté interprétative :

Un texte est littéraire, selon la logique proposée par Fish, dans la mesure où il est utilisé de façon littéraire. Il n'est donc pas littéraire « en soi », il peut seulement le devenir (en fonction de l'usage qu'on en fait). [...] la littérarité n'est pas à chercher dans le texte lui-même (ce qui entérinerait une distinction d'essence ou de nature entre textes littéraires et des textes non-littéraires); la littérarité résulte de la projection sur le texte d'un certain type d'approche auquel on le soumet en vue d'en tirer certains effets $^{(114)}$.

La fable de Stanley Fish, comme d'ailleurs la pratique de nombreux écrivains, témoigne donc de ce que tout texte peut être littérarisé, dans la mesure où il est l'objet d'un usage littéraire. D'autre part, fait remarquer Yves Citton, la théorie du lecteur-faiseur de textes révèle que tout objet peut devenir un instrument d'oppression ou d'émancipation, selon l'usage que l'on en fait :

Autrement dit : tout objet a le potentiel de devenir une bombe à retardement. Cela ouvre le champ à une conception de la politique très différente de celle qui domine dans la France d'aujourd'hui - une politique qui n'est à penser ni en termes d'essence, d'être ou d'action, (modèle « jacobin »), ni en termes d'identité (modèle « communautariste »), mais en terme de devenirs, de transformations, de réappropriations créatrices, de détournements imprévisibles et de piratages enjoués ${ }^{(115)}$.

\section{Les apports de Stanley Fish}

Il est urgent, pour finir, d'esquisser un bilan et de se demander quel est le gain que les études littéraires, et en particulier les critiques et les lecteurs, peuvent espérer, aujourd'hui d'une (re)découverte des théories de Stanley Fish. Il est légitime de se demander si elles ouvrent des pistes prometteuses, par leur applicabilité, pour ceux qui mènent des analyses empiriques, même si ce dernier se plaît à répéter que son entreprise de théorisation a peu de répercussion - sinon aucune - sur les méthodologies et les résultats de l'analyse concrète des textes ${ }^{(116)}$.

Tout d'abord, la lecture de Stanley Fish permet de rompre avec une certaine grisaille qui règne actuellement, en France, dans la critique littéraire universitaire. En effet, il est de bon ton, avec un regard de soulagement et un petit sourire moqueur et entendu, de se féliciter de la fin de l'ère terroriste des théoriciens qui auraient sévi dans les années soixante et soixante-dix et de l'obsolescence - « on vous l'avait bien dit !»-de leurs délires spéculatifs, et de constater, par un retour heureux du balancier ${ }^{(117)}$, un retour vers le bon sens du néo-positivisme qui appelle des études sérieuses, humbles, concrètes et fondées sur des faits objectifs, comme ceux que repèrent et décrivent l'histoire littéraire, la philologie, la linguistique et la stylistique, enfin débarrassée de ses frénésies

(114)Ibid., p. 23 ; souligné par l'auteur.

(115)Ibid., pp. 23-24; souligné par 1'auteur.

(116) Voir, à cet égard, la postface de Stanley Fish, dans laquelle - ultime provocation ? - il semble défendre une critique fondée sur l' «intention de l'auteur », tout en précisant qu'elle est impénétrable.

(117) Voir à ce sujet Antoine Compagnon, «Introduction. Que reste-il de nos amours? », in Le Démon de la théorie. Littérature et sens commun, op. cit., pp. 11-13. 
structuralistes. En effet, à rebours, il y a chez Stanley Fish une jouissance de la spéculation théorique qui produit une véritable bouffée d'air qui revigore. Car comme le fait remarquer très justement Yves Citton, dans sa préface, un tel recours à l'affabulation théorique, même si elle peut paraître parfois prétentieuse, voire arrogante, fascine « en ce qu'elle émane d'un enjouement [...] qui tient surtout à la joie (exubérante et contagieuse) de jouer le jeu de la théorie [...] et au plaisir presque sensuel que prend l'auteur à raconter des fables théoriques, et à considérer la pensée comme un lieu d'expérimentations presque jubilatoire ${ }^{(118)}$. " C'est cette même allégresse intellectuelle que l'on retrouve, pour prendre deux exemples paradigmatiques, dans les travaux de Marc Escola et de Pierre Bayard, tous deux attentifs aux devenirs, aux transformations, aux réappropriations créatrices, aux détournements imprévisibles et aux piratages enjoués ${ }^{(119)}$ des textes lus et étudiés. Ainsi, Marc Escola, dans Lupus in fabula ${ }^{(120)}$, procède à une « approche résolument ludique des Fables de La Fontaine »; il ne propose pas " une nouvelle interprétation, mais l'élaboration d'un mode de lecture, librement inspiré des jeux sérieux de l'Oulipo comme des théories sur la réécriture, qui suppose au sein d'une fable la production imaginaire de "fables possibles" "; en effet, étant donné que « la loi même du genre veut qu'il y ait toujours dans une fable de quoi en faire une autre, [et que] chaque fable [est] elle-même le produit d'une réécriture, » le lecteur se livre « à son tour à un exercice de transformation "; " "affabuler" les fables, c'est donc à la fois réfléchir sur les choix de La Fontaine entre plusieurs versions possibles de ses textes sources, et esquisser en retour quelques apologues nouveaux ; c'est aussi et surtout se rendre sensible à la "fabrique" même du dispositif fabulaire »; bref, il s'agit de rêver ou d'écrire « « les fables fantômes qui hantent les fables réelles, ce qui nous permet de découvrir un La Fontaine finalement inédit tout à la fois plus "gaillard" et plus politique que celui consacré par la tradition scolaire. » De la même façon, Pierre Bayard, à la façon de Stanley Fish ${ }^{(121)}$, prend un grand plaisir - et en donne à son lecteur - à imaginer des affabulations théoriques assez surprenantes qui bousculent les évidences, communément admises, sur le lecteur et sur les textes. Ainsi n'hésite-t-il pas à refaire et à déconstruire l'enquête bâclée d'Hercule Poirot pour démasquer l'assassin probable (le vrai ?) de Roger Ackroyd qu'aurait caché, et pour de bonnes raisons, le narrateur d'Agatha Christie. Son iconoclastie joyeuse, qui sait jouer sur le paradoxe et les implications théoriques qui en découlent, n'en reste pas là dans les différents livres qu'il publie et on peut relever, pour illustration, quelques chantiers ouverts par Pierre Bayard ${ }^{(122)}$ : il reprend l'enquête sur la mort du père d'Hamlet, et fait le constat que les différentes interprétations ne parlent pas, en fait, du même texte et que donc il ne peut exister qu'un dialogue de

(118) $Q L F$., Yves Citton, «Puissance des communautés interprétatives », pp. 15-16.

(119) Je reprends les termes d'Yves Citton, ibid., p. 24.

(120) Marc Escola, Lupus in fabula. Six façons d'affabuler La Fontaine, Paris, Presses universitaires de Vincennes, 2003. Je reprends, ici, la présentation du livre qui est faite sur le site Fabula, URL : http ://www.fabula.org/actualites/marc-escola-lupus-in-fabula-six-facons-d-affabuler-lafontaine_6486.php; consulté le 12 juillet 2011.

(121)Si Stanley Fish (communauté interprétative) et Pierre Bayard (paradigme intérieur) se rejoignent dans la reprise et l'utilisation qu'ils font de la notion de paradigme, développée par Thomas Kuhn dans son étude sur l'histoire des sciences (La Structure des révolutions scientifiques, 1962, trad. de l'allemand par L. Meyer, Paris, Flammarion, coll. « Champs », 1983), il faut noter que les positionspropositions de Pierre Bayard (voir les distinctions qu'il opère entre livre collectif, livre intérieur individuel, livre-écran, livre réel, livre fantôme) permettent de tempérer, à bon escient, les aspects les plus anti-subjectifs et anti-idiosyncrasiques que peuvent prendre les thèses de Stanley Fish - et en particulier l'affirmation de l'autorité sans partage des communautés interprétatives.

(122)Pierre Bayard, respectivement aux Éditions de Minuit, coll. "Paradoxe ": Qui a tué Roger Ackroyd?,1998; Comment améliorer les æuvres ratées?, 2000 ; Enquête sur Hamlet. Le Dialogue de sourds, 2002 ; Demain est écrit, 2005 ; Comment parler des livres que l'on n'a pas lus ?, 2007 ; Le Plagiat par anticipation, 2009 ; Et si les æuvres changeaient d'auteur ?, 2010 ; je reprends, succinctement, les informations données sur la $\mathrm{C} 4$ de ces livres qu'on peut raisonnablement attribuer à l'auteur. 
sourds entre les différents interprètes qui sélectionnent unilatéralement les marques textuelles qui conviennent à leur thèse, en passant, allègrement, sous silence celles qui fragiliseraient leurs arguments; partant du constat que les plus grands écrivains ont connu des moments de faiblesses et ont raté certaines de leurs œuvres (histoires aberrantes, personnages inconsistants, style déplorable...), il pose la question de la création et du génie et propose des améliorations concrètes qui permettent d'imaginer, entre rêve de perfection et délire de réécriture, ce que les œuvres auraient pu être dans des contextes littéraires différents; il pose, exemples à l'appui, la question de savoir si la littérature peut prédire l'avenir, puiser une partie de son inspiration dans le futur, ce qui, en cas de réponse positive, nous inciterait à raconter la vie des écrivains en commençant par la fin ; il explique comment on peut parler des livres qu'on n'a pas lus avec pertinence, et peut-être mieux, si l'on suit le conseil d'Oscar Wilde qui, non sans humour, proclamait qu'il refusait de lire les livres dont il faisait la critique, par peur de se laisser influencer ; il détecte les plagiats par anticipation en envisageant l'influence qu'exercent les écrivains et les artistes les uns sur les autres à rebours de la logique temporelle (Voltaire, par exemple, plagiant Conan Doyle), ce qui l'amène à souhaiter une réécriture de l'histoire de la littérature, en mettant en évidence les véritables filiations, débarrassées des contraintes et des limites de la chronologie ; enfin, il n'hésite pas, dans un pur esprit borgésien ${ }^{(123)}$, à procéder à de fausses attributions d'auteurs (Tolstoï, par exemple devenant l'auteur d'Autant en emporte le vent), en mettant en évidence le bénéfice que l'on pourrait tirer d'une telle opération qui permettrait de découvrir les œuvres sous un angle inhabituel et original.

De telles affabulations théoriques ont certes le pouvoir salutaire de nous délurer par rapport à la doxa ambiante, grâce à leur entrain ludique, contre-intuitif et disruptif ; mais, elles ouvrent, aussi, des voies pour penser autrement les études littéraires et les grandes questions qui ne cessent de les hanter : Pourquoi lisons-nous? Qu'est-ce qu'un texte ? Pourquoi, à quelles conditions et comment lire littérairement certains textes ? Quels effets et quelles transformations attendons-nous, nous les lecteurs-faiseurs de textes, de nos interprétations littéraires ? Quelle est notre part de liberté, de créativité dans l'interprétation des textes? À quelles communautés interprétatives appartenonsnous et comment gérons-nous leurs contraintes ? Comment pouvons-nous concilier - ou non- les contradictions éventuelles que cette pluri-appartenance peut engendrer? En vue de quel(s) usage(s) lisons-nous ? En quoi nos lectures se connectent-elles avec nos formes de vie, avec les autres discours qui nous traversent, avec nos expériences quotidiennes? Leur force et leur utilité consistent précisément à remettre en cause - en tous les cas à les questionner - nos évidences, nos idées reçues, notre vocabulaire fondamental, dirait Richard Rorty. S'interroger sur elles ne revient pas à estimer, du point de vue de Sirius, leur degré de vérité - tâche vaine, on l'a vu -, mais, dans une perspective pragmatiste, de voir dans quelle mesure elles transforment en mieux notre rapport à la littérature et les usages que nous en faisons. C'est ainsi que nous deviendrons - et que nous formerons - des lecteurs ironistes, pour qui « il n'y a jamais que des contextes ».

(123) Voir J.-L. Borges, « Pierre Ménard, auteur du Quichotte», in Fictions, 1944, Paris, Gallimard, Folio, 1974. 